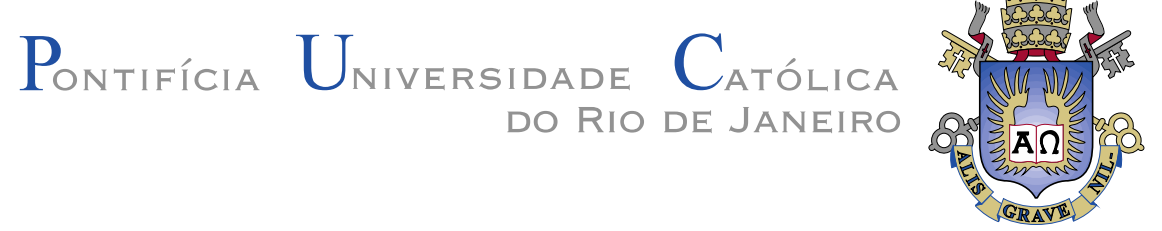

Renata Fortini Moustafá Osman

\title{
Processamento e Caracterização de Nanocompósitos de Nylon 11 Reforçados por Nanotubos de Titanatos Modificados por Surfactantes
}

\begin{abstract}
Dissertação de Mestrado
Dissertação apresentada como requisito parcial para obtenção do grau de Mestre pelo Programa de Pós-graduação em Engenharia de Materiais e de Processos Químicos e Metalúrgicos do Departamento de Engenharia de Materiais do Centro Técnico Científico da PUC-Rio.
\end{abstract}

Orientador: Prof. Fernando Cosme Rizzo Assunção

Co-Orientador: Prof. Bojan A. Marinkovic

Rio de Janeiro

Abril de 2013 


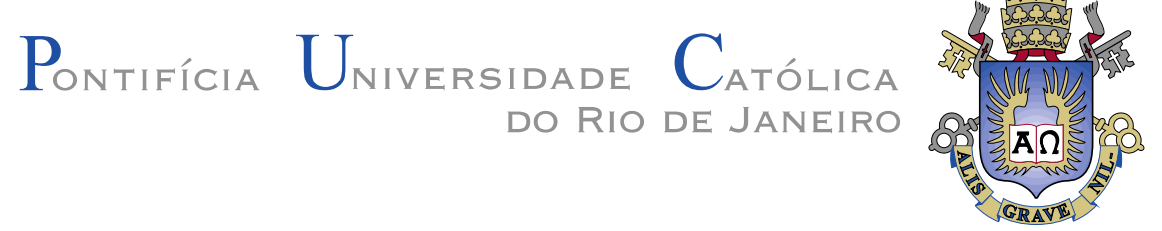

Renata Fortini Moustafá Osman

\section{Processamento e Caracterização de Nanocompósitos de Nylon 11 Reforçados por Nanotubos de Titanatos Modificados por Surfactantes}

Dissertação apresentada como requisito parcial para obtenção do grau de Mestre pelo Programa de Pósgraduação em Engenharia de Materiais e de Processos Químicos e Metalúrgicos do Departamento de Engenharia de Materiais do Centro Técnico Científico da PUC-Rio.

Prof. Fernando Cosme Rizzo Assunção

Orientador

Departamento de Engenharia de Materiais - PUC-Rio

Prof. Bojan Marincovic

Co-Orientador

Departamento de Engenharia de Materiais - PUC-Rio

Profa. Paula Mendes Jardim

UFRJ

Prof. Marcos Henrique de Pinho Maurício

Departamento de Engenharia de Materiais - PUC-Rio

Prof. José Eugenio Leal

Coordenador Setorial do Centro Técnico Científico - PUC-Rio 
Todos os direitos reservados. É proibida a reprodução total ou parcial do trabalho sem autorização da universidade, da autora e do orientador.

\section{Renata Fortini Moustafá Osman}

Engenheira de Materiais formada na Pontifícia Universidade Católica do Rio de Janeiro, 2010.

Ficha Catalográfica

Osman, Renata Fortini Moustafá

Processamento e caracterização de nanocompósitos de Nylon 11 reforçados por nanotubos de titanatos modificados por surfactantes / Renata Fortini Moustafá Osman ; orientador: Fernando Cosme Rizzo Assunção ; coorientador: Bojan A. Marinkovic. - 2013.

110 f. : il. (color.) ; $30 \mathrm{~cm}$

Dissertação (mestrado)-Pontifícia Universidade Católica do Rio de Janeiro, Departamento de Engenharia de Materiais, 2013.

Inclui bibliografia

1. Engenharia de materiais - Teses. 2. Nanotubos de titanato. 3. Surfactantes. 4. Propriedades mecânicas. 5. Nylon 11. 6. Compósitos I. Assunção, Fernando Cosme Rizzo. II. Marinkovic, Bojan A. III. Pontifícia Universidade Católica do Rio de Janeiro. Departamento de Engenharia de Materiais. IV. Título.

CDD: 620.11 


\section{Agradecimentos}

Aos meus orientadores Prof. Rizzo e Bojan, que sempre me apoiaram, incentivaram para que meus objetivos fossem cumpridos e acreditaram em meu potencial.

À PUC-Rio, pelos auxílios concedidos, sem os quais este trabalho não poderia ter sido realizado. À Petrobras por ter oferecido esta bolsa de estudos e esta oportunidade

À Patricia, que com toda a sua sabedoria, persistência e perfeccionismo me mostrou os caminhos certos para o desenvolvimento de uma dissertação de mestrado.

Ao aluno de Iniciação Científica Gerson, pois sem sua força física e dedicação eu jamais teria conseguido fabricar os meus compósitos.

À Slavica que em tão pouco tempo se tornou uma grande amiga e companheira de trabalho.

Ao Victor que teve muita paciência ao ouvir meus desabafos.

Ao Luis Carlos, simplesmente por existir.

À minha mãe, simplesmente por ser a mãe que sempre quis.

A todos que me ajudaram direta ou indiretamente, Juliana, Yuri, Arnon, Aline, Marcos, Márcia, Lidija, Leandro, Mauricio, Antonio Mario, Antonio Gutierrez, Marco Abreu, Prof. Zé Roberto, Prof. Avillez, etc. 


\section{Resumo}

Osman, Renata Fortini Moustafá; Assunção, Fernando Cosme Rizzo. Processamento e caracterização de nanocompósitos de Nylon 11 reforçados por nanotubos de titanatos modificados por surfactantes. Rio de Janeiro, 2013, 110p. Dissertação de Mestrado - Departamento de Engenharia de Materiais, Pontifícia Universidade Católica do Rio de Janeiro.

Processamento e Caracterização de Nanocompósitos de Nylon 11 reforçados por Nanotubos de Titanatos Modificados com Surfactantes estuda a influência da adição de nanotubos de titanato (TTNTs) nas propriedades mecânicas do Nylon 11 (Poliamida 11). De acordo com estudos prévios, espera-se que pequenas quantidades de TTNTs melhorem propriedades como resistência mecânica e rigidez. No entanto, a incorporação dessas nanopartículas também pode acarretar numa piora dessas propriedades, caso não haja uma dispersão eficaz na matriz polimérica. Assim sendo, também foram avaliados compósitos de Nylon 11 e nanotubos de titanato modificados com 2 tipos de surfactantes, um catiônico (CTAB) e outro aniônico (SDS) para investigação do efeito destes surfactantes na dispersão dos TTNTs e por consequência nas propriedades finais dos compósitos comparados ao Nylon 11 puro. Foi realizado também um estudo da dispersão dos nanotubos com e sem mistura com surfactante em água e butanol, em três intervalos de tempo, 15, 30 e 60 min a fim de se escolher os melhores parâmetros para a dispersão. Foram produzidos compósitos em uma microextrusora e uma microinjetora. Os compósitos com TTNTs tratados com CTAB apresentaram aumentos de $15 \%$ e $10 \%$ no módulo de elasticidade e os compósitos com TTNTs tratados com SDS apresentaram aumentos de $26 \%$ e $20 \%$ para essa mesma propriedade, respectivamente nas concentrações de 0.5 e $2 \%$ wt de TTNTs, em relação ao Nylon 11 puro.

\section{Palavras-chave}

Nanotubos de titanato; surfactantes; propriedades mecânicas; Nylon 11; compósitos. 


\section{Abstract}

Osman, Renata Fortini Moustafá; Assunção, Fernando Cosme Rizzo (Advisor). Processing and characterization of Nylon 11 nanocomposites reinforced with titanates nanotubes modified by surfactants. Rio de Janeiro, 2013, 110p. MSc. Dissertation Departamento de Engenharia de Materiais, Pontifícia Universidade Católica do Rio de Janeiro.

Processing and Characterization of Nylon 11 Nanocomposites Reinforced with Titanates Nanotubes Modified by Surfactants has investigated the effect of titanate nanotubes (TTNTs) in the mechanical properties of Nylon 11 (polyamide 11) polymer. According to the existing literature small amounts of TTNTs in polymer matrix improve properties such as tensile strength and stiffness. However, the addition of these nanoparticles may also lead to a decrease of strength of the composite if the nanotubes are not well dispersed in the polymer matrix. Therefore, Nylon 11 composites with TTNTs treated with 2 kinds of surfactant - a cationic (CTAB) and an anionic (SDS) - were also investigated. The surfactants act as dispersion agents leading to a better dispersion state of nanotubes and as a final result, promote enhancement of composites' mechanical properties. Also, the dispersion of nanotubes was studied with and without mixing surfactants in distilled water and butanol, in three different time intervals (15, 30 and 60 minutes) to assess the optimal dispersion parameters. Composites were produced in a microextruder and a microinjector. The composites with TTNTs treated with CTAB displayed an increase of $15 \%$ and $10 \%$ in the Young's Modulus and the composites with TTNTs treated with SDS displayed an increase of $26 \%$ and $20 \%$ in the same property, respectively in the concentrations of 0.5 and $2 \%$ wt of TTNTs, compared to pure Nylon 11.

\section{Keywords}

Titanate nanotubes; surfactants; mechanical properties; Nylon 11; composites. 


\section{Sumário}

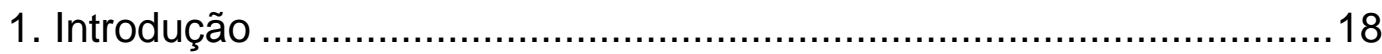

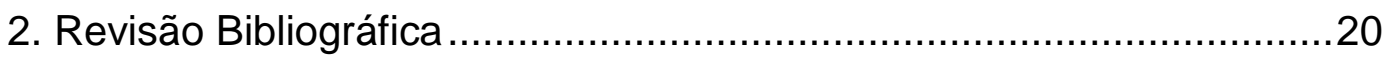

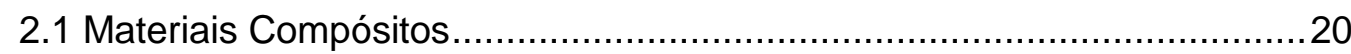

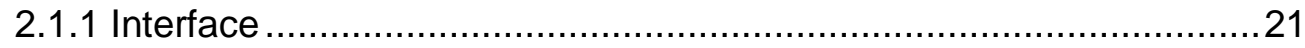

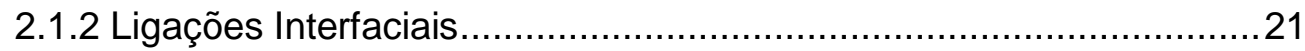

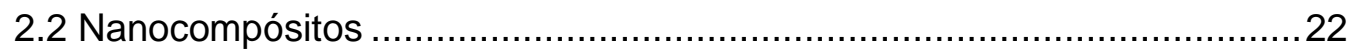

2.2.1 Propriedades mecânicas de nanocompósitos de

matriz polimérica termoplástica e partículas inorgânicas............................23

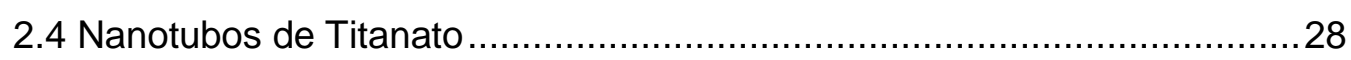

2.4.1 Fabricação de titânia e titanatos nanoestruturados

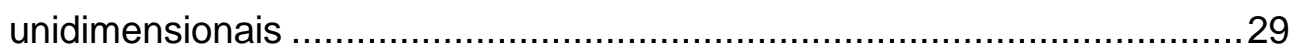

2.4.2 Estrutura e morfologia dos nanotubos de titanato...............................30

2.4.3 Propriedades mecânicas de compósitos de matriz polimérica e nanotubos de titanato ……………………..................30

2.5 Modificação da superfície com surfactantes..............................................33

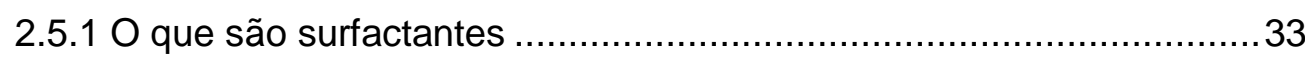

2.5.2 Adsorção de surfactantes e a modificação da interface......................35

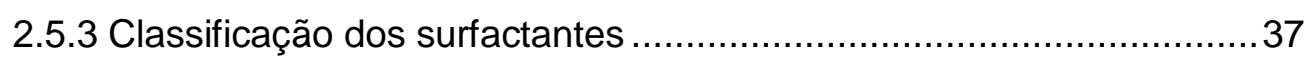

2.5.4 Como atuam as forças elétricas na superfície das partículas .............37

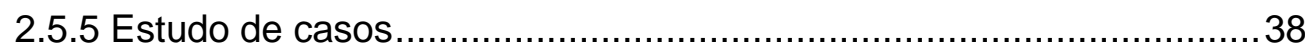

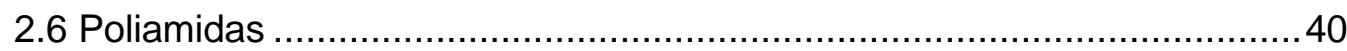

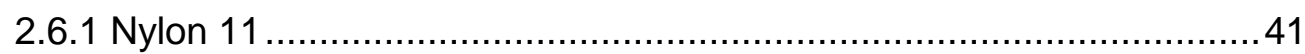

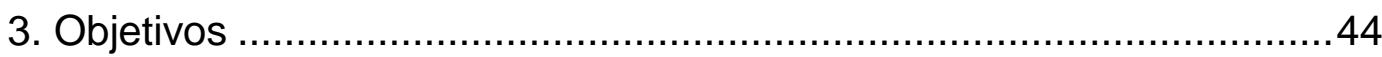

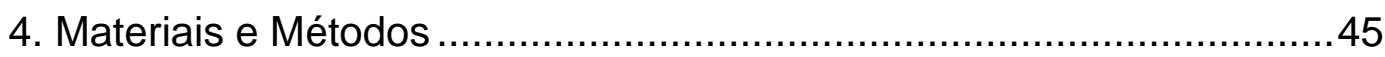

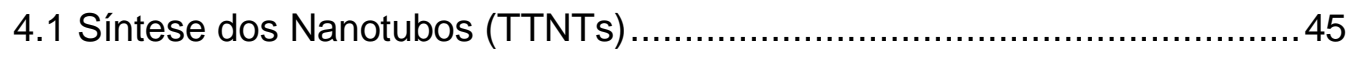

4.2 Dispersão dos TTNTs com surfactantes ...............................................46

4.2.1. Definição da concentração de surfactantes e

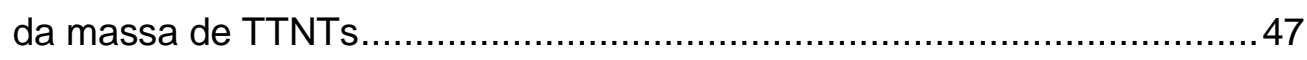

4.3 Caracterização dos TTNTS puros e tratados com surfactantes.................49

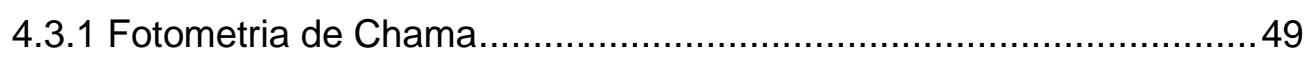

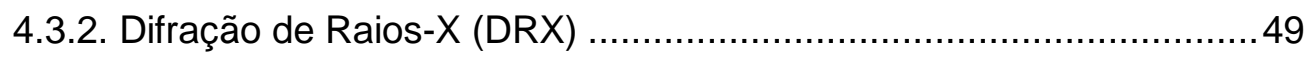

4.3.3. Microscopia eletrônica de transmissão (MET) …………………...... 49

4.3.4. Microscopia eletrônica de varredura (MEV) ....................................5

4.3.5 Medições de área superficial específica ...........................................50 


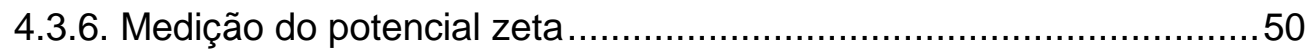

4.3.7 Análise Termogravimétrica (TGA) ……...................................51

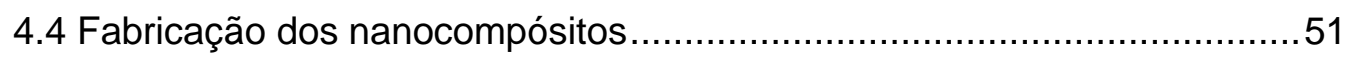

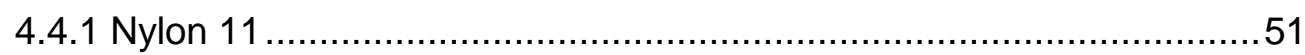

4.4.2. Processamento dos nanocompósitos de Nylon 11 .........................52

4.5. Caracterização dos nanocompósitos..................................................54

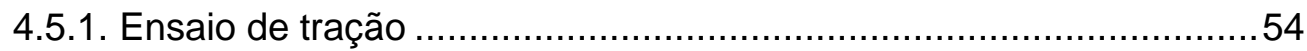

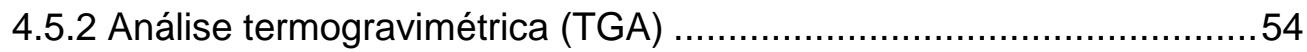

4.5.3. Calorimetria diferencial de varredura (DSC) …..............................55

4.5.4 Microscopia eletrônica de varredura (MEV) .....................................55

5. Resultados e Discussão .......................................................... 56

5.1 Caracterização dos nanotubos de titanato puros ……............................56

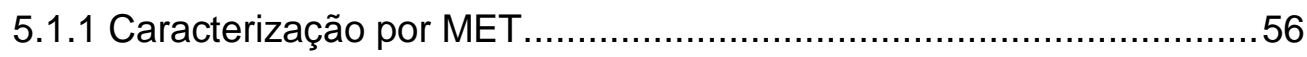

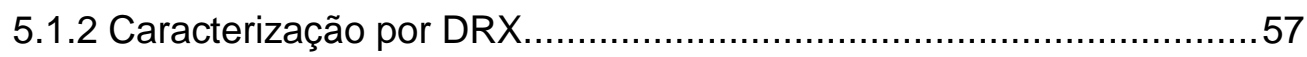

5.1.3 Análise da área superficial específica..........................................59

5.1.4 Análise do potencial zeta......................................................60

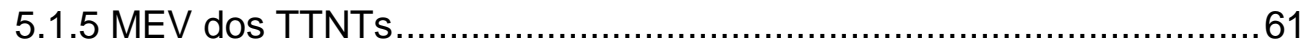

5.2 Estudo da dispersão dos nanotubos com surfactantes ..........................62

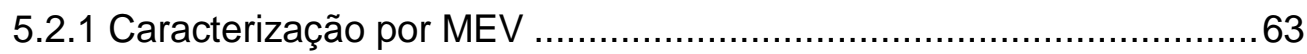

5.3 Caracterização dos TTNTs após o tratamento com surfactantes .............69

5.3.1 MEV dos TTNTs com surfactantes ..............................................69

5.3.2 TGA dos TTNTs com surfactantes ........................................... 72

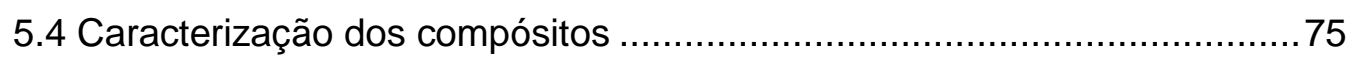

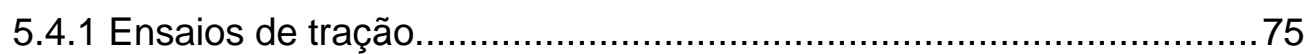

5.4.2 Análise da superfície de fratura dos compósitos .............................87

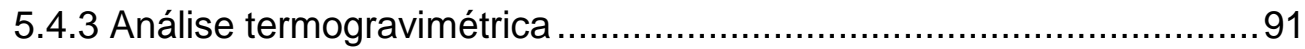

5.4.4 Calorimetria diferencial de varredura..........................................93

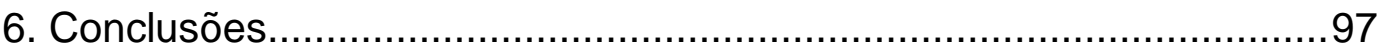

7. Referências Bibliográficas .........................................................100

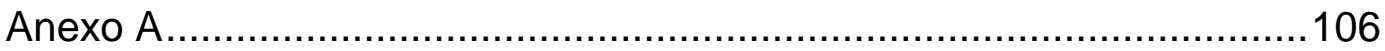




\section{Lista de Figuras}

Figura 1: Compósitos com reforço na forma de particulados

(a), fibras curtas (b) e fibras longas (c) [3] 20

Figura 2: Ligações Interfaciais: Ancoragem mecânica

(a); Atração eletrostática (b)

22

Figura 3: Nanomateriais (OD e 1D) e suas possíves dimensões. 23

Figura 4: Imagens de MEV (a) de um aglomerado de nanopartículas de TiO2 com uma interação de repulsão com o polímero ao redor e (b) um agrupamento de nanopartículas de TiO2 modificado com interações de atração com o PMMA [21] 26

Figura 5: Imagens de MET de CNTs (a) sem tratamento com surfactante; (b) com $1 \mathrm{CMC}$ de Triton $\mathrm{x}$ e (c) com $10 \mathrm{CMC}$ de Triton $\mathrm{x}$. 27

Figura 6: Gráfico da resistência à flexão versus concentração de CNTs para compósitos com e sem tratamento com Triton X [22] ......27

Figura 7: Gráfico do módulo de flexão versus concentração de CNTs para compósitos com e sem tratamento cm Triton X [22]. 28

Figura 8: Imagem de MEV da superfície de fratura de um compósito com $1 \%$ em peso TTNTs [30]

Figura 9: Morfologia dos TTNTs após os diferentes processos

de secagem: $(a, d)$ secagem ao ar, $(b, e)$ secagem à vácuo e (c, f) secagem por liofilização. A linha de cima apresenta 
imagens de FESEM e a de baixo imagens de MET [15] ........................32

Figura 10: Estrutura esquemática de um surfactante [32] ......................34

Figura 11: Esquema da atuação do CTAB ao ser misturados a

TTNTs [33] 34

Figura 12: llustração do processo de micelização do surfactante

Dodecil Sulfato de Sódio (SDS) em solução aquosa [39] 35

Figura 13: Ângulo de contato e tensões superficiais entre as três

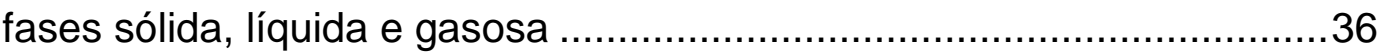

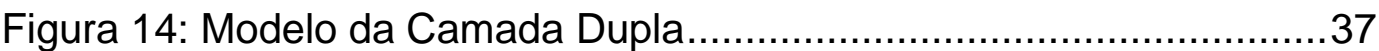

Figura 15: Potencial Zeta das soluções de nanotubos de titanato com diferentes quantidades de CTAB a pH 9.5 40

Figura 16: Monômeros da poliamida 6 e da poliamida 6,6 41

Figura 17: Monômero do Nylon 11 41

Figura 18: Seção de um tubo flexível e suas diversas camadas 42

Figura 19: Curva Tensão x Deformação para o Nylon 11 e os compósitos de nanoargila em diversas concentrações [20] 43

Figura 20: Reator Berghof usado na síntese dos TTNTs 45

Figura 21: Procedimento padrão para o estudo da dispersão de TTNTs 48

Figura 22: Microextrusora utilizada para a fabricação dos compósitos ....52 Figura 23: Microinjetora utilizada para a fabricação dos compósitos 53

Figura 24: Formato do corpo de prova fabricado na microextrusora e na microinjetora. 
Figura 25: Imagens de MET: (a, c, e) TTNTs/H; (b, d, f) TTNTs/L ..........57

Figura 26: Difratograma da amostra TTNTs/H. .................................58

Figura 27: Difratograma da amostra TTNTs/L.....................................58

Figura 28: Gráfico do potencial zeta para as amostras TTNTs/H

e TTNTs/L

Figura 29: Imagens de MEV no modo de elétrons secundários

dos TTNTs/H com aumentos de 500, 2000, 5000 e 15000x.

Figura 30: Imagens de MEV no modo de elétrons secundários dos TTNTs/H dispersos em butanol e água após 15, 30 e 60 min. Aumento de 33x. 64

Figura 31: Imagens de MEV no modo de elétrons secundários dos TTNTs/H dispersos com CTAB em butanol e água após 15, 30 e $60 \mathrm{~min}$. 66

Figura 32: Imagem de MEV no modo de elétrons secundários dos TTNTs/L dispersos com SDS em butanol e água após 15, 30 e $60 \mathrm{~min}$.

Figura 33: Imagens de MEV no modo de elétrons secundários das melhores dispersões obtidas. 68

Figura 34: Imagens de MEV no modo de elétrons secundários da comparação entre as amostras TTNTs, TTNTs/CTAB e TTNTs/SDS. 70

Figura 35: Imagem de MEV no modo de elétrons secundários de TTNTs após dispersão com SDS em água por 30 min. 
Figura 36: Imagem de MEV com a demarcação dos pontos

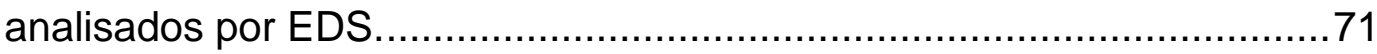

Figura 37: Gráficos obtidos com a análise EDS...................................72

Figura 38: Curva termogravimétrica e derivada da

curva termogravimétrica para a amostra TTNTs/H.

Figura 39: Curva termogravimétrica e derivada da

curva termogravimétrica para a amostra TTNTs/CTAB.

.73

Figura 40: Curva termogravimétrica e derivada da

curva termogravimétrica para a amostra TTNTs/L.

74

Figura 41: Curva termogravimétrica e derivada da

curva termogravimétrica para a amostra TTNTs/SDS

Figura 42: Gráfico da tensão no escoamento versus \% em peso de

TTNTs.

Figura 43: Gráfico da deformação no escoamento versus \% em peso

de TTNTs 78

Figura 44: Gráfico da tensão na ruptura versus \% em peso de

TTNTs.

Figura 45: Gráfico da deformação na ruptura versus \% em peso de

TTNTs.

Figura 46: Gráfico do módulo de elasticidade versus \% em peso

de TTNTs.

Figura 47: Imagens de MEV no modo de elétrons secundários

da microestrutura da fratura do Nylon 11: (a) região central da 
fratura; (b) e (c) ampliação da região da vértice da fratura.

Figura 48: Imagem de MEV no modo de elétrons secundários da fratura de um compósito de Nylon $11 \mathrm{com} 1 \%$ em peso de TTNTs/H e os respectivos aumentos (b, c). 88

Figura 49: Imagens de MEV no modo de elétrons secundários da superfície de fratura do compósito de Nylon 11 com $0.5 \%$ em peso de TTNTs/CTAB. 89

Figura 50: Imagens de MEV no modo de elétrons secundários da superfície de fratura do compósito de Nylon 11 com $0.5 \%$ em peso de TTNTs/SDS. 90

Figura 51: Imagem de MEV no modo de elétrons secundários de um aglomerado de TTNTs/SDS em meio à matriz de Nylon 11. Imagem da fratura .91

Figura 54: Curvas térmicas de DSC do Nylon 11 94

Figura 55: Curvas térmicas de DSC do compósito com $2 \%$ em peso de TTNTs/L 95

Figura 56: Gráfico da tensão de escoamento versus \% em peso de TTNTs com barras de erro. 106

Figura 57: Gráfico da deformação no escoamento versus \% em peso de TTNTs com barras de erro. 106

Figura 58: Gráfico da tensão de ruptura versus \% em peso de TTNTs com barras de erro. 107

Figura 59: Gráfico da deformação na ruptura versus \% em peso de 
TTNTs com barras de erro.

Figura 60: Gráfico do módulo de elasticidade versus \% em peso de TTNTs com barras de erro. 108

Figura 59: Curva obtida com a análise termogravimétrica para o

Nylon 11 puro. 108

Figura 60: Curva obtida com a análise termogravimétrica

para o compósito de Nylon 11 com 2\% em peso de TTNTs/CTAB........109 


\section{Lista de tabelas}

Tabela 1: Definição da concentração dos surfactantes .........................47

Tabela 2: Relação dos parâmetros da dispersão ................................48

Tabela 3: Propriedades gerais do Nylon 11 fornecidas pela

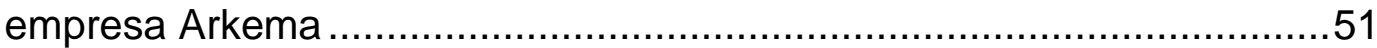

Tabela 4: Compósitos produzidos na extrusora de acordo com a

amostra de nanotubos utilizada como reforço e as concentrações

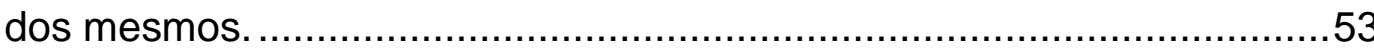

Tabela 5: Área superficial específica das amostras TTNTs/H e

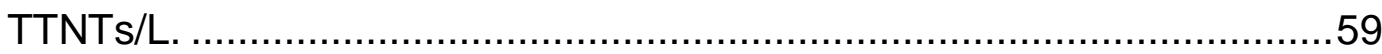

Tabela 6: Valores de PIE do TTNTs/H e TTNTs/L obtidos com a

medição do potencial zeta........................................................60

Tabela 7: Parâmetros da dispersão dos TTNTs...............................69

Tabela 8: Descrição dos compósitos e suas respectivas

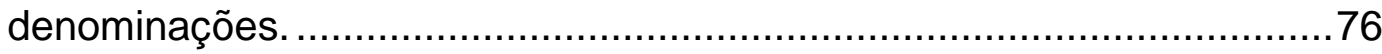

Tabela 9: Resultados da tensão de escoamento em relação ao

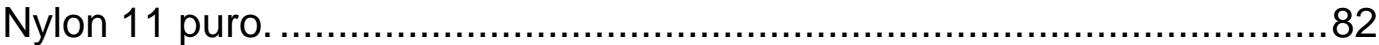

Tabela 10: Resultados da deformação no escoamento em

relação ao Nylon 11 puro.

Tabela 11: Resultados da tensão ne ruptura em relação ao

Nylon 11 puro.

Tabela 12: Resultados da deformação na ruptura em relação ao

Nylon 11 puro 
Tabela 13: Resultados do módulo de elasticidade em relação ao

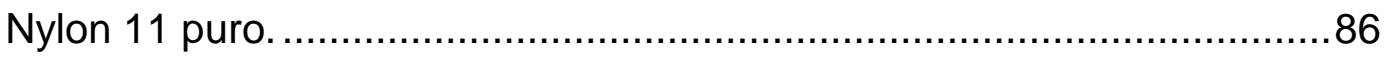

Tabela 14: Amostras analisadas por TGA............................................92

Tabela 15: Resultados das análises termogravimétricas, em

ordem crescente de temperatura de degradação...................................93

Tabela 16: Melhores resultados obtidos na análise por TGA e

aumento da temperatura de degradação em relação ao Nylon 11 ...........93

Tabela 17: Relação da $T_{g}$ obtida para cada material, em ordem

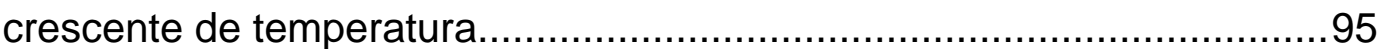




\section{Lista de abreviações}

BET Brunauer, Emmett e Teller, autores da metodologia

CNT Nanotubos de carbono

CTAB Brometo de cetiltrimetilamônio (surfactante)

DSC Calorimetria diferencial de varredura

DRX Difração de raios $X$

MEV Microscopia eletrônica de varredura

MET Microscopia eletrônica de transmissão

TGA Análise termogravimétrica

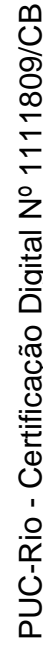

TTNTs Nanotubos de Titanato

TTNTs/H Nanotubos de Titanato com alto teor de sódio

TTNTs/L N Nanotubos de Titanato com baixo teor de sódio

TTNTs/CTAB Nanotubos de titanato tratados com o surfactante CTAB

TTNTs/SDS Nanotubos de titanato tratados com o surfactante SDS

$\mathrm{T}_{\mathrm{g}} \quad$ Temperatura de transição vítrea

SDS Dodecil sulfato de sódio (surfactante) 